\title{
Minnesota's Early Experience with Medical Home Implementation: Viewpoints from the Front Lines
}

\author{
Patricia Fontaine, $M D, M S^{7}$, Robin Whitebird, $P h D, M S W^{7}$, Leif I. Solberg, $M D^{7}$, Juliana Tillema, MPA , \\ Angela Smithson, $\mathrm{MD}^{2}$, and Benjamin F. Crabtree, $P h D^{3}$
}

'HealthPartners Institute for Education and Research, Minneapolis, MN, USA; ${ }^{2}$ Department of Family Medicine and Community Health, St John's Hospital Family Medicine Residency, University of Minnesota, St. Paul, MN, USA; ${ }^{3}$ Department of Family Medicine and Community Health, Division of Research, Rutgers Robert Wood Johnson Medical School, New Brunswick, NJ, USA.

BACKGROUND: Evidence is evolving about the impact of patient-centered medical homes (PCMHs) on important outcomes in primary care. Minnesota has developed its own PCMH certification process, envisioned as an all-payer initiative with an emphasis on patientcenteredness, which may add unique experiences and outcomes to the national discussion.

OBJECTIVE: We aimed to identify the facilitators and barriers encountered by nine diverse primary care practices selected from the first 80 to achieve PCMH certification in Minnesota.

DESIGN: This was a qualitative analysis of semistructured, in-person interviews.

PARTICIPANTS: Thirty-one administrative and clinical leaders, including clinic managers, physician champions, medical directors, nursing supervisors, and care coordinators participated in the study.

KEY RESULTS: Six factors emerged as most important to the efforts to become PMCHs: leadership support, organizational culture, finances, quality improvement (QI) experience, information technology (IT) resources, and patient involvement. Facilitators included committed leadership at local and higher levels, prior experience and ongoing support for QI initiatives, and adequate financial and IT resources. Reimbursement was a significant barrier due to perceived inadequacy and inconsistent participation by health plans. The unsuitability of electronic medical records (EMRs) to PCMH documentation requirements likewise presented ongoing challenges. Many interviewees described patient input as helpful to their clinics' $\mathrm{PCMH}-$ related changes and were enthusiastic about their "patient partners." The majority of interviewees felt that becoming a PCMH was right for patients and was personally worthwhile, even while acknowledging the tremendous effort involved and voicing skepticism about reimbursement over the short term.

CONCLUSIONS: The experience of participants in Minnesota's state-wide initiative to legislate PCMH transformation provides a broad view of facilitators and barriers. Unique facilitators included a requirement for patient involvement, which pushed practices to create patient-centered innovations, and new reimbursement models based on quality indicators for a population.

Received April 21, 2014

Revised October 16, 2014

Accepted November 14, 2014

Published online December 13, 2014
Among barriers were the costs to practices and patients, and EMRs that failed to accommodate PCMH requirements.

J Gen Intern Med 30(7):899-906

DOI: $10.1007 / \mathrm{s} 11606-014-3136-y$

(C) Society of General Internal Medicine 2014

\section{INTRODUCTION}

The Agency for Healthcare Research and Quality (AHRQ) defines the patient-centered medical home (PCMH) as a model structure for primary health care that is patient-centered, comprehensive, and coordinated, with accessible services and a commitment to quality and safety. ${ }^{1}$ First described by the American Academy of Pediatrics in 1967, the PCMH was endorsed by all four major U.S. primary care professional associations in 2007. ${ }^{2}$ Since then, the PCMH has evolved rapidly from a theoretical construct into real-world implementation, with $26 \mathrm{PCMH}$ demonstration projects under way in 18 states by $2010 .^{3}$ Research evaluating the PCMH's effectiveness in achieving the goals of improved care quality, patient experience, and reduced healthcare costs has encouraged further refinement of the model. ${ }^{4-11}$ Facilitators and barriers to the creation of a successful PCMH have been explored in a limited number of heterogeneous settings, including small practices, large group practices, high-performing practices, and safety net clinics. ${ }^{12-17}$ To date, significant challenges have been identified; specifically, the investment of resources for infrastructure changes and the leadership skills needed to guide an organization through paradigm-shifting cultural change. ${ }^{5,14,18}$ Common facilitators have included valuing innovation, quality improvement, and patient-centeredness. ${ }^{12,13,16}$

We sought to augment the literature on motivations, barriers, and facilitators to $\mathrm{PCMH}$ implementation by describing a large-scale initiative with some unique features.

Minnesota developed its PCMH certification standards in response to a legislative mandate, using a multi-stakeholder process that involved representatives from provider, payer, and patient advocacy organizations. Although standards mirrored the basic tenets of PCMH Recognition from the National Committee on Quality Assurance, ${ }^{19}$ unique features included 
1) a site visit by trained individuals from Minnesota Department of Health; 2) promotion of patient involvement in quality improvement activities; and 3) a goal to have all payers in the state participate.

\section{METHODS}

\section{Study Design}

This is a qualitative study of the first practices certified as PCMHs in Minnesota. Qualitative research can illuminate processes and attitudinal factors that would not be gleaned from quantitative data alone, and is therefore well suited to describing complex real-world phenomena such as the $\mathrm{PCMH}^{20}$

\section{Setting}

In 2008, as a part of state health reform legislation, the Minnesota Departments of Health and Human Services were charged with developing a voluntary certification process for Healthcare Homes (HCHs), Minnesota's term for PCMHs. Certification standards were published in 2010 and included stipulations for: 1) access and communication with the patient and family; 2) a searchable registry to manage services and identify care gaps; 3 ) care coordination; 4) care plans created with patients; and 5) a continuous improvement process that included a quality-improvement committee with active patient recruitment and participation. ${ }^{21}$. Primary care clinics that met $\mathrm{HCH}$ standards through a written application and site visit became eligible for a per-patient-per-month care coordination fee (CCF), in addition to fees for services. However, the CCF could only be applied to patients with complex and chronic medical conditions who chose to enroll in the HCH. The Minnesota Department of Human Services established the CCF for government-subsidized health plans based on three defined tiers of complexity, with the highest payment for the most complex patients. Private insurers negotiated CCFs with the state.

\section{Sample Selection}

In 2010 and 2011, 157 Minnesota clinics achieved HCH certification. From the first 80 that were certified, we purposively sampled ten clinics for the in-depth interviews that would represent diversity of size, location, medical group affiliation and PCMH attainment. For PCMH attainment, we created a composite score based on 1) scores for diabetes and cardiovascular outcomes from the mandatory Statewide Quality Measurement Reporting System (http://www. mnhealthscores.org); and 2) a practice systems score from the Physician Practice Connection-Research Survey (PPCRS), which rates the presence of clinical systems important for PCMH success. We chose five clinics with the highest scores and five with the lowest scores, replacing with the next-ranked clinic as necessary to maximize diversity of geography, size, ownership and practice type. Nine clinics agreed to participate. We asked clinics to select individual participants who were familiar with the PCMH concept and had direct experience with their clinic's $\mathrm{HCH}$ certification process. We suggested including a physician leader, clinic manager, care coordinator, and one or two other care team members. Some higher-level administrators were also chosen because they had worked closely with the clinic during the process.

\section{Data Collection}

Two investigators trained in qualitative interviewing conducted semi-structured in-person interviews with two to six participants at each clinic over three months in 2011. The interview script consisted of a verbal assent process followed by 11 open-ended questions and follow-up probes. (Appendix A, available online). Study investigators created questions to elicit motivations for becoming a PCMH, barriers and facilitators encountered, and whether the process had been worthwhile. We piloted the questions, and used them in the field with no further revisions. Each of the nine practices received a $\$ 2,000$ honorarium. A local institutional review board approved the study protocol.

\section{Data Analysis}

All interviews were audio taped and professionally transcribed. Five study team members, including the two interviewers, independently reviewed each transcript and coded key factors. The team met regularly, and using a constant comparative method, ${ }^{22}$ developed a final coding structure. We used Microsoft Excel spreadsheets to organize coded data into six key factors identified from multiple interviews. We analyzed the facilitators and barriers within each factor and identified any differences according to composite score. Finally, we compiled responses to the question, "Was it worth the effort for your clinic to become a medical home?"

\section{KEY RESULTS}

We interviewed 21 women and ten men at nine primary care clinics (Table 1), including one family medicine residency. Mean duration of the interviews was 33 minutes (range, 1262 minutes). Similar to the larger sample from which they were chosen, two were independent clinics, while the others were affiliated with larger medical groups within health systems. Clinics represented different geographic locations (three each of urban, suburban/small city, and rural).

From the interviews, we identified six factors with key roles in clinics' PCMH change processes: leadership, culture, finances, quality improvement (QI), information technology (IT), and patient involvement. We report the interviewees' perspectives on these factors, and Table 2 summarizes the facilitators or barriers most relevant to each. 
Table 1. Clinic Demographics

\begin{tabular}{|c|c|c|c|c|c|}
\hline Clinic & Location & Affiliation & \# MDs & Specialty & Interviews \\
\hline A & Urban & Large medical group & 8 & Pediatric & $2=\mathrm{PL} / \mathrm{CM}$ \\
\hline B & Urban & Large MG/health system & 17 & Internal medicine & $4=\mathrm{PL} / \mathrm{CM} / \mathrm{CC} / \mathrm{CC}$ \\
\hline $\mathrm{C}$ & Rural & Large MG/health system & 4 & Family medicine & $3=\mathrm{PL} / \mathrm{CM} / \mathrm{CC}$ \\
\hline $\mathrm{D}$ & Urban & Large MG/health system & 5 & Family medicine residency & $3=\mathrm{PL} / \mathrm{CM} / \mathrm{CM}$ \\
\hline $\mathrm{E}$ & Rural & Independent, rural health system & 11 & Family medicine & $4=\mathrm{PL} / \mathrm{DCS} / \mathrm{CC} / \mathrm{CFO}$ \\
\hline $\mathrm{F}$ & Urban & Large MG/health system & 16 & Primary care & $6=\mathrm{PL}(3) / \mathrm{CM} / \mathrm{CC} / \mathrm{CNS}$ \\
\hline $\mathrm{G}$ & Rural & Independent & 4 & Family medicine & $3=\mathrm{PL} / \mathrm{CM} / \mathrm{CC}$ \\
\hline $\mathrm{H}$ & Urban & Large MG/health system & 13 & Primary care & $3=\mathrm{PL} / \mathrm{CM} / \mathrm{CDS}$ \\
\hline I & Suburb & Large MG/health system & 4 & Primary care & $3=\mathrm{PL} / \mathrm{CM} / \mathrm{CC}$ \\
\hline
\end{tabular}

CC care coordinator (pediatric or adult) or patient care manager; $C F O$ chief financial officer; CDS care-delivery supervisor; CM clinic manager; CNS clinic nursing supervisor; DCS director of clinical services; MG medical group; PL physician leader or champion, medical director, or assistant medical director

Primary care $=$ family medicine, internal medicine, and pediatrics

\section{Leadership Support}

At all clinics, regardless of size or transformation score, interviewees made a strong case for organizational leadership and commitment "right up to the CEO." They consistently described how leaders with strong vision, enthusiasm, and passion facilitated change, "a new generation of leaders now having the opportunity to mold primary care." (Physician Leader, PL)

At the clinic level, a respected physician champion, especially one with support from all leadership levels, was sometimes cited as the most important factor for success. There appeared to be a special synergy at clinics in which administrators and clinicians shared a vision and goals. "In our organization, we have a dyad leadership model whereby the physicians and administrators partner and that is the fundamental core of success." (Clinic Manager, CM)

Finally, interviewees noted that leaders with the power of the purse strings could direct resources to the $\mathrm{HCH}$ effort, including paid, protected time for physicians and care coordinators.

Table 2. Key Factors Involved in Patient-Centered Medical Home (PCMH) Transformation

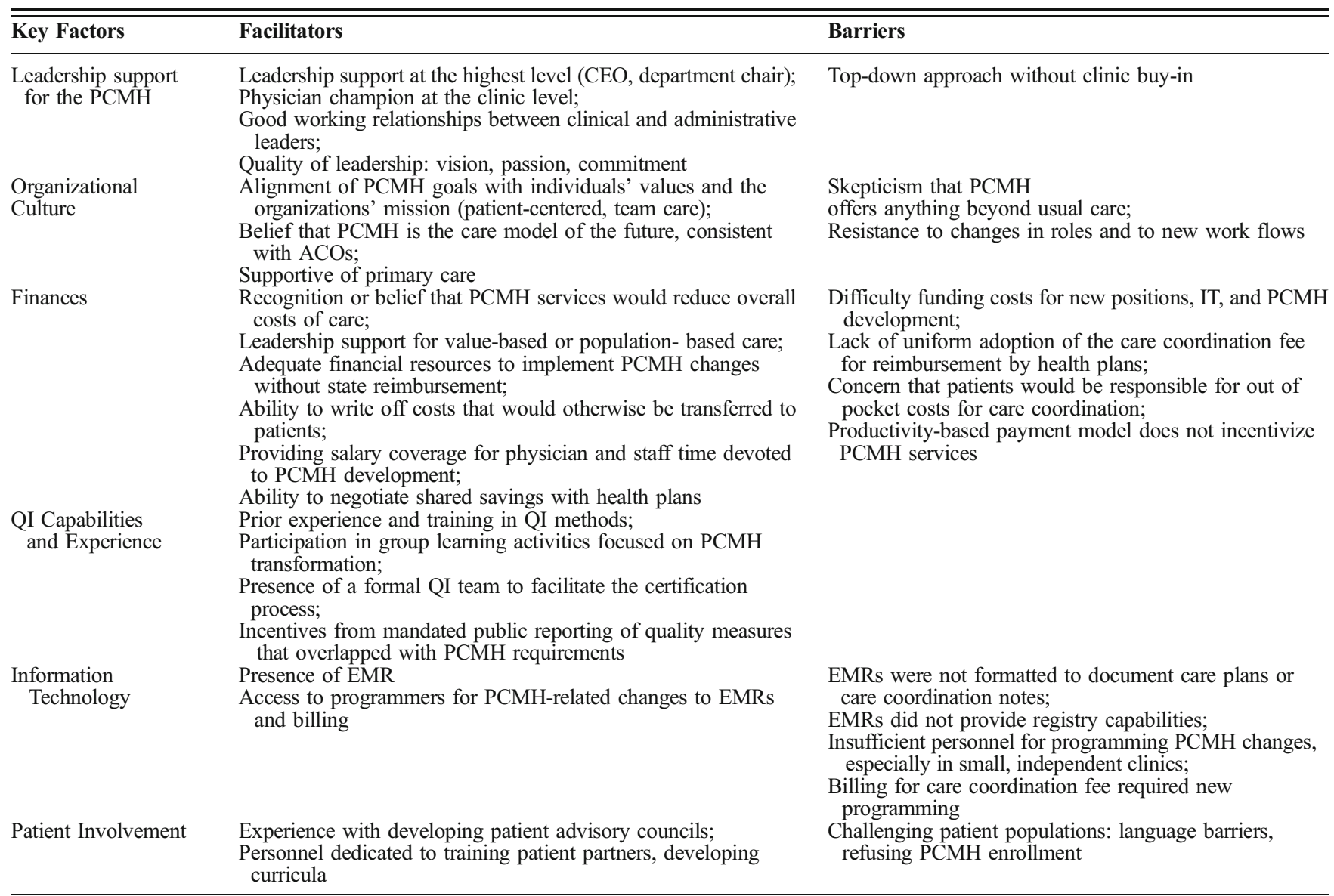


Interviewees did not often specify leadership barriers; however, a top-down leadership decision to pursue $\mathrm{HCH}$ certification left one practice's interviewees feeling unengaged: "It wasn't our clinic that made an individual decision to do this. I'm not sure we would have come up with this process." (PL)

\section{Organizational Culture}

Interviewees described their perceptions of their clinic's or system's values and recognized the importance of alignment between PCMH goals and the organization's mission: "The goals of the medical home certification process in Minnesota were an excellent match with our own...especially the patient centeredness and the team-based care, which are both longstanding traditions [here], although they definitely needed some reinterpretation for the 21st Century." (PL)

A prominent cultural value was high-quality care, which often equated to being patient-centered. "Our system as a whole is focused on patient-centered and family-centered care. That is, in a nutshell, what healthcare home is. " (Care coordinator, CC) The practice with the highest PCMH attainment score emphasized a "passion for patient care" and described how "the essence of medical home is relationship building." (PL)

Innovation was another important value. In the residency clinic, innovation meant "training [our residents] in the newest and latest things.” (CM) In larger systems, PCMHs were seen as "stepping stones" to accountable care organizations (ACOs): "Most of [our PCMH] work evolved because our health system really wants to achieve ACO status...because an ACO is basically a very functional medical home that has a [broader] network." (PL)

A culture valuing primary care also emerged as a facilitator. "[Deciding to be a medical home] was really about how to keep physicians in primary care." (CM) "I think you just have to see that the system has to change. A...specialty-based system of healthcare can't survive." (PL) Others recognized the PCMH as a welcome return to fundamental primary care values: "I think another important factor is that we started out with physicians who are all family practice residency-trained. So if you get down to their roots...they were all trained in this concept." (PL)

Barriers included resistance to culture change and skepticism. Care coordinators cited difficulties with "physicians who think, 'I'm already doing this for my patients.", and the "significant change in culture and expectations to go to team care," where individuals "practiced at the top of their licenses" and the "right people were doing the right work." Some nurses "felt like it took some responsibilities away from them," while physicians asked "Am I gonna take a hit in my pay because of this?"(CM) Clinics with higher attainment scores were not exempt: "It's a big culture change. As always, there are people who want to do it, those who come along kicking and screaming, and those who don't come along at all who are still fussing." (PL)

\section{Finances}

Interviewees spoke frequently about financial issues. Although the promise of a CCF was an incentive for some practices to become medical homes, barriers quickly emerged: start-up costs, difficulties with reimbursement, misalignment of existing clinic pay structures with PCMH goals, and negative impacts on patients. "Of the top ten [barriers], the first nine are cost... money invested in jobs...in policy development...IT changes... and people having to travel to meetings." (PL) One care coordinator said: "The monthly [reimbursement] is...not real hefty, and the level doesn't necessarily reflect the complexity of working with that patient."

In addition, interviewees voiced displeasure that, while the state paid the CCFs for patients with subsidized health insurance, commercial health plans were slow and inconsistent in doing so. "It's a crime in the state that [the $\mathrm{HCH}$ ] is only covered by some insurance and not others." (PL) A care coordinator described how "[reimbursement] has been painful, problematic; frustrating...It's very hard with an individual patient to find out if we will be paid." Some practices found that "families [were] getting billed every month to pay out of pocket for care coordination, and [couldn't] see the benefit because it's abstract and not what they've experienced [before]." (PL)

Most interviewees agreed that the prevailing model for payment to physicians, based on productivity or relative value units (RVUs), was another barrier. They clearly saw how volume-based, per-visit reimbursement models failed to incentivize the enhanced, patient-centered care envisioned for PCMHs. "As long as you're paid on RVUs, care-planning meetings and team meetings have to be high yield and not super intrusive on your time...Because you know you have to produce [the RVUs you] contracted to produce." (PL)

Interviewees at two practices with high scores described innovative approaches to overcoming financial barriers. In one health system, "[The governing board] told us to focus on the Triple Aim... and not be so concerned about financial measures that show profitability on volumes." (PL) In the second, "the highest leadership took [providers] off the 'RVU treadmill'. They no longer look [only] at [primary care] visits...it's emergency room visits and specialist consults... and [the total] cost to take care of the population." (PL) Further, "[providers are] paid a certain percentage on their quality and patient satisfaction ... and that's very new." (CM)

To simplify billing and protect patients from co-payment, some practices decided to forego state reimbursement: "We don't bill any of our patients [for care coordination]. Whichever payers are willing to reimburse us, we bill them. But if there's any patient liability remaining, we don't pass that on to them." (CFO)

Several interviewees described how administrators protected salaries to overcome physician resistance (e.g., "for one year during the transition to PCMH," "half time for our physician champion," "comp time for getting 
involved"). Larger organizations found niches in their budgets to pay care coordinators. Several practices sought supplemental PCMH grant funding, and one had dedicated revenue from affiliation with a critical access hospital. One independent clinic negotiated for sharing savings with a health plan after finding "a $40 \%$ savings among patients in our HCH." (CM)

\section{Quality Improvement Experience and Capabilities}

A background of exposure to formal QI experiences was perceived as setting the stage for PCMH improvements. Many practices had participated in QI activities with, for example, the Institute for Clinical Systems Improvement (ICSI), Institute for Healthcare Improvement (IHI), or Institute for Patientand Family-Centered Care. Experience with QI initiatives provided training in chronic disease management and careimprovement strategies that later supported PCMH changes. "We'd been working ... long before the HCH certification process came into play, going back to the mid-nineties in activities with ICSI and IHI. We actually had our first family medicine diabetes coordinator in 1998. So things we have been striving to do for years suddenly are becoming possible as we move into the medical home era." (PL) "We started looking at chronic disease across the system over five years ago...I worked on diabetes, and another team worked on congestive heart failure, and then we realized it needed to be broader ...embedded in our primary care clinics." (Care coordination supervisor)

Interviewees appreciated learning collaboratives where sharing strategies and success stories with other practices helped participants identify barriers and address challenges. "I can call $W$ clinic, which has already been doing this a year and a half, and say, 'What do you think about this? What have you done?"' (CC) "[We] were involved in the pediatric medical home learning collaborative... and it was a transformational experience. It's hard to overestimate how good that was. " $(\mathrm{CM})$

QI resources, personnel, and administrative infrastructure were also cited as important facilitators. Clinics that were part of larger systems had internal QI teams that led the approach to transformation: "We started with our [health system's] QI team presenting to us: 'we're looking at becoming $\mathrm{HCH}$ certified.' It was proposed as a new way of caring for a patient." (Nursing supervisor, NS) "QI provided us with what we needed to do to become certified. They took that huge document that was tough to read [the certification standards] and narrowed it down." (CM) "I leaned on [our health system's quality team] for support through the entire process." (PL)

Minnesota's HCH requirements to maintain registries and engage in continuous QI activities dovetailed well with mandatory participation in the existing statewide quality measurement reporting system (SQRMS). Outcomes for diabetes, asthma, and other conditions required by SQRMS naturally became an important focus for $\mathrm{HCH}$ QI activities. Shared reports led to friendly competition among clinics. "I noticed at one of the other sites, their numbers for optimal care for asthma went up significantly. So I asked them what they were doing, and they told me. So I tried [what they had done] and bam, our numbers went up!" (CM)

No clinic described barriers related to QI factors. However, two of the five clinics with lower scores provided only one coded QI comment, giving the impression that the absence of comments was itself relevant: specifically, that practices with QI experience and resources recognized the value, while those without did not register QI as an issue.

\section{Information Technology}

Interviewees acknowledged the pivotal role of IT in PCMH infrastructure. "You have to have IT resources in order to give you infrastructure for a care plan, a registry, care coordination notes...." (PL) Even so, IT was most often cited as a barrier to PCMH transformation. Both the format of existing electronic medical records (EMRs) and the availability of knowledgeable IT staff were widely seen as problematic. "It was a huge thing, getting IT to make our EMR do what we needed to do, collect information not only for [HCH requirements] but also for [the statewide quality reporting system]." (CM) Even clinics with the highest $\mathrm{n}$ scores described an "ongoing struggle," while a small, independent clinic experienced an IT "nightmare" when they had to reprogram customized PCMH features by hand due to an EMR upgrade: "My 'IT Department' is two people in the basement who, in addition to doing everything for the hospital, have to do special things for our EMR in the clinic. We had to create all new fields in our electronic records." (PL)

New programming was also required to capture reimbursement for HCH CCFs, creating burden "because [IT staff] have to write a whole new system of billing for those charges." (PL) Clinics from larger health systems were more likely to have access to programmers who could make changes to the EMR; however, that did not eliminate problems: "All the work is very important, it's just, 'Who's going to get that IT resource first?' It's a common theme I hear from other clinics. We have limited IT resources." (PL)

\section{Patient Involvement}

Involving patients in their care and clinic operations was typically described in positive terms: "patient input is key...a big part of why it works," and "a very positive thing... kind of fun." One practice had "formed patient and family advisory councils many years ago," and more recently "developed a 'Patients as Partners' program, hired a coordinator, [and] developed training modules for patients as advisors, as mentors, and...how to tell their stories." (PL) However, not all practices were equally experienced or successful in engaging patients. Another "had to build a list of patient partners... 
starting with no one...going to each provider for good candidates." (CC) The care-delivery manager in one large clinic without a patient advisory group observed, "A barrier we still have is how to engage a patient... you don't want to mention the word 'program.' It turns people off." Patient populations with language barriers, difficulty paying for visits, or cultural differences leading to lack of trust in the medical system were cited as particularly challenging to engage.

Finally, interviewees described how patients' positive experiences with the PCMH increased their own job satisfaction and trust in the process. "The first patient... who called in and said, 'I' $m$ a healthcare home patient and I need to talk to the care coordinator,'...To see that click is the payoff." (CM)

\section{Would They Do It Again?}

At the conclusion of the semi-structured interview, each person was asked, "Was it worth the effort for your clinic to become a medical home?" Eighteen of 31 interviewees were unequivocally positive, citing improvements in patient care, clinic care processes, and job satisfaction: "I'd do it again in a heartbeat. It has been the career-changing thing in my life." (CC) "It's worth the effort because patients are happier, getting better care." (CC) "We thought it would increase the rate of change [improvement] here...definitely found that to be true."(PL) Those who gave an equivocal response (12 of 31) often recognized benefits but were not convinced about the tradeoffs. "It's definitely the right work for the patient. I don't know that we're seeing the reimbursement for the hard work we're doing." (CM) Only one interviewee replied, "No. And that's real disappointing." (PL)

\section{DISCUSSION}

Our findings from Minnesota's early experiences with a unique statewide PCMH certification process provide new insights about facilitators and barriers to PCMH transformation while echoing other national pilots in some respects.

Patient participation was a requirement of Minnesota's $\mathrm{HCH}$ certification standards that pushed practices to develop patient advisory councils and innovative ways to identify, engage, and train "patient partners." Compared with previous reports that only $29 \%$ of 112 PCMHs had patients as advisors, and only $32 \%$ provided consistent opportunities for patients to participate in QI, ${ }^{23} 100 \%$ of Minnesota $\mathrm{HCHs}$ reported actively recruiting patients for their clinic QI committees. This reflects the power of a legislative mandate. Also notable was the personal satisfaction and career-renewing energy several of our interviewees reported as a result of working directly with patient partners. Future research should evaluate the sustainability and long-term impacts of patient involvement in PCMHs.
Our interviewees cited the misalignment between traditional volume-based pay structures and PCMH goals as a key barrier to change, reinforcing findings from others ${ }^{24,25}$ who describe reimbursement as "one of the most important policy problems affecting implementation of $\mathrm{PCMH}, " 24$ and conclude that health systems should reward primary care physicians for achieving quality measures on population-based care. ${ }^{3}$ We found evidence that population-based models for physician compensation are being cautiously adopted in some high-functioning Minnesota PCMHs.

We also heard how not all payers covered the care coordination fee, leaving some patients responsible for a monthly payment. This unanticipated outcome arose because the state covered care coordination fees for patients on Medicaid and Medicare and assumed that private plans would do the same. However, the 2008 healthcare reform legislation could not enforce compliance. Private plans elected instead to treat the fee as a cost that would be borne by patients, and some practices responded by forgoing reimbursement for care coordination to prevent patients from being charged. This unsustainable business model should be addressed by payment reform legislation or health system policies. ${ }^{26,27}$

Similar to findings from other studies, ${ }^{14,15,18}$ clinical and administrative leadership proved a crucial facilitator at the clinic level and higher. Our interviewees universally endorsed the need for leaders who were 1) inspiring and 2) able to effect fundamental operational changes. ${ }^{14}$ The importance of facilitative leadership fits well with the concept of adaptive reserve (i.e., combination of healthy relationship infrastructure, alignment of management systems, and leadership), which Nutting et al. observed as critical to managing practice change. ${ }^{28}$

Minnesota has a high penetration of EMRs in primary care. ${ }^{29}$ This study showed that having an EMR was less of an issue than having the personnel and resources to modify it for PCMH purposes. The lack of fields to express concepts such as a care plan, for example, was frustrating and burdensome to practices. For this situation to improve, vendors must incorporate patient-centeredness and coordination of care into the next generation of EMRs. ${ }^{30}$

Our study has limitations as well as strengths. Although we devised a clinic selection process for diversity, our sample of early adopters does not represent all primary care practices, particularly practices outside Minnesota. There may be biases among practices motivated to achieve PCMH status early, although it is uncertain what direction such biases might take. Likewise, our findings of more QI capabilities and innovative reimbursement models among clinics with higher PCMH attainment scores must be interpreted with caution, given our small sample. Finally, our research team attempted to minimize personal biases by acknowledging them and using group consensus to identify key factors. 
Finally, it is noteworthy that all but one of our interviewees said that becoming a PCMH was worth it, and many expressed positive views emphatically: "If you could have heard the eloquence with which [our patient partners] spoke about the lift of the burden of disease, anxiety and worry... and the good care that they felt they were getting [from] this model as opposed to traditional care, you would never again question if it was worth it." (PL)

Acknowledgements: The authors gratefully acknowledge Marie Maes-Voreis, Director, Health Care Homes at the Minnesota Department of Health $(\mathrm{MDH})$ for manuscript review, Ann Harste for manuscript preparation, Mary VanBeusekom for editing assistance, and the members of the Healthcare Home Research Advisory Council who represented their clinics as part of the study team.

Funding: This research was funded by AHRQ grant \#1R18HSO19161.

Conflict of interest: The authors declare no conflict of interest.

Corresponding Author: Patricia Fontaine, MD, MS; HealthPartners Institute for Education and Research, PO Box 1524, MS 23301A, Minneapolis, MN 55440-1524, USA (e-mail: Patricia.L.Fontaine@ HealthPartners.Com).

\section{REFERENCES}

1. Agency for Healthcare Research and Quality. Defining the PCMH. Rockville, MD: Agency for Healthcare Research and Quality; [cited 2014 November 19]; Available from: http://pcmh.ahrq.gov/page/definingpcmh.

2. American Academy of Family Physicians (AAFP), American Academy of Pediatrics (AAP), American College of Physicians (ACP), American Osteopathic Association (AOA). Joint Principles of the Patient-Centered Medical Home. Washington DC2007 [cited 2014 November 19]; Available from: http://www.acponline.org/running_practice/delivery_and_payment_ models/pcmh/demonstrations/jointprinc_05_17.pdf.

3. Bitton A, Martin C, Landon BE. A nationwide survey of patient-centered medical home demonstration projects. J Gen Intern Med. 2010;25(6):584592.

4. Peikes D, Zutshi A, Genevro JL, Parchman ML, Meyers DS. Early evaluations of the medical home: building on a promising start. Am J Manage Care. 2012;18(2):105-116.

5. Crabtree BF, Nutting PA, Miller WL, Stange KC, Stewart EE, Jaen CR. Summary of the National Demonstration Project and recommendations for the patient-centered medical home. Ann Fam Med. 2010;8(Suppl 1):S80S90. S2.

6. Homer CJ, Klatka K, Romm D, Kuhlthau K, Bloom S, Newacheck $\mathbf{P}$, et al. A review of the evidence for the medical home for children with special health care needs. Pediatrics. 2008;122(4):e922-e937.

7. Fields D, Leshen E, Patel K. Analysis \& commentary. Driving quality gains and cost savings through adoption of medical homes. Health Aff (Millwood). 2010;29(5):819-826.

8. Nielsen M, Langner B, Zema C, Hacker T, Grundy P. Benefits of implementing the primary care patient-centered medical home: A review of cost and quality results, 2012. Washington: Patient-Centered Primary Care Collaborative; 2012

9. Raskas RS, Latts LM, Hummel JR, Wenners D, Levine H, Nussbaum SR. Early results show WellPoint's patient-centered medical home pilots have met some goals for costs, utilization, and quality. Health Aff (Millwood). 2012;31(9):2002-2009.
10. Solberg LI, Crain AL, Tillema J, Scholle SH, Fontaine P, Whitebird R. Medical home transformation: a gradual process and a continuum of attainment. Ann Fam Med. 2013;11(Suppl 1):S108-S114.

11. Friedberg MW, Schneider EC, Rosenthal MB, Volpp KG, Werner RM. Association between participation in a multipayer medical home intervention and changes in quality, utilization, and costs of care. JAMA. 2014;311(8):815-825.

12. Scholle SH, Asche SE, Morton S, Solberg LI, Tirodkar MA, Jaen CR. Support and strategies for change among small patient-centered medical home practices. Ann Fam Med. 2013;11(Suppl 1):S6-S13.

13. Dohan D, McCuistion MH, Frosch DL, Hung DY, Tai-Seale M. Recognition as a patient-centered medical home: fundamental or incidental? Ann Fam Med. 2013;11(Suppl 1):S14-S18.

14. McMullen CK, Schneider J, Firemark A, Davis J, Spofford M. Cultivating engaged leadership through a learning collaborative: lessons from primary care renewal in Oregon safety net clinics. Ann Fam Med. 2013;11(Suppl 1):S34-S40.

15. Rittenhouse DR, Schmidt LA, Wu KJ, Wiley J. Incentivizing primary care providers to innovate: building medical homes in the post-Katrina New Orleans safety net. Health Serv Res. 2014;49(1):75-92.

16. McAllister JW, Cooley WC, Van Cleave J, Boudreau AA, Kuhlthau K. Medical home transformation in pediatric primary care-what drives change? Ann Fam Med. 2013;11(Suppl 1):S90-S98.

17. Gabbay RA, Friedberg MW, Miller-Day M, Cronholm PF, Adelman A, Schneider EC. A positive deviance approach to understanding key features to improving diabetes care in the medical home. Ann Fam Med. 2013;11(Suppl 1):S99-S107.

18. Donahue KE, Halladay JR, Wise A, Reiter K, Lee SY, Ward K, et al. Facilitators of transforming primary care: a look under the hood at practice leadership. Ann Fam Med. 2013;11(Suppl 1):S27-S33.

19. National Committee for Quality Assurance. Patient-centered medical home recognition. Washington, DC: NCQA; 2014 [cited 2014 November 19]; Available from: http://www.ncqa.org/Programs/Recognition/Practices/ PatientCenteredMedicalHomePCMH.aspx.

20. Bradley EH, Curry LA, Devers KJ. Qualitative data analysis for health services research: developing taxonomy, themes, and theory. Health Serv Res. 2007;42(4): 1758-1772.

21. Minnesota Department of Health. Certification \& Recertification Requirements and Process. St. Paul, MN2014 [cited 2014 November 19]; Available from: http://www.health.state.mn.us/healthreform/homes/certification/ index.html.

22. Bernard HR, Ryan HW. Analyzing Qualitative Data: Systematic Approaches. Thousand Oaks: Sage Publications, Inc; 2010.

23. Han E, Hudson Scholle S, Morton S, Bechtel C, Kessler R. Survey shows that fewer than a third of patient-centered medical home practices engage patients in quality improvement. Health Aff (Millwood). 2013;32(2):368-375.

24. Alexander JA, Cohen GR, Wise CG, Green LA. The policy context of patient-centered medical homes: perspectives of primary care providers. J Gen Intern Med. 2013;28(1):147-153.

25. Bitton A, Schwartz GR, Stewart EE, Henderson DE, Keohane CA, Bates Dw, et al. Off the hamster wheel? Qualitative evaluation of a paymentlinked patient-centered medical home (PCMH) pilot. Milbank $\mathrm{B}$. 2012;90(3):484-515.

26. Rajkumar R, Conway PH, Tavenner M. CMs—engaging multiple payers in payment reform. JAMA [Internet]. 2014 [cited 2014 November 19]. Available from: http://dx.doi.org/10.1001/jama.2014.3703.

27. Gauthier A, Cullen A. Reforming health care delivery through payment change and transparency: Minnesota's innovations. Washington DC: The Commonwealth Fund and the National Academy for State Health Policy; 2010 [cited 2014 November 19]; Available from: http://www. commonwealthfund.org/publications /fund-reports /2010/mar/ reforming-health-care-delivery-through-payment-change-and-transparency-minnesotas-innovations.

28. Nutting PA, Crabtree BF, Miller WL, Stewart EE, Stange KC, Jaen CR. Journey to the patient-centered medical home: a qualitative analysis of the experiences of practices in the National Demonstration Project. Ann Fam Med. 2010;8(Suppl 1):S45-S56. S92.

29. Fontaine P, Zink T, Boyle RG, Kralewski J. Health information exchange: participation by Minnesota primary care practices. Arch Intern Med. 2010;170(7):622-629.

30. Leventhal T, Taliaferro JP, Wong $\mathbf{K}$, Hughes C, Mun S. The patientcentered medical home and health information technology. Telemed $\mathrm{J}$ Health Off J Am Telemed Assoc. 2012;18(2):145-149. 


\section{APPENDIX A: KEY INFORMANT INTERVIEW QUESTIONS}

Clinic Name:

Interviewee Name:

Role:

Date:

Interview Questions

1. We're interested in why your practice decided to become a patient-centered medical home. Could you tell me about that?

Note- separate plan to become a medical home from plan to be certified as a $\mathrm{HCH}$

Probe Areas

- MDH certification

- Reimbursement

- Practice re-design

$2 \mathrm{~A}$. What types of changes did you need to make to become a medical home?

2B. Can you tell me about the problems you faced in making these changes?

Probe Areas

- Technical changes (i.e., personnel, processes, job descriptions, information tools)

- Clinic culture

- Attitudes

- Resources

- Strategies for achieving change

3A. Can you tell me what facilitated your clinic becoming a medical home?

3B. What factors were most important or most helpful in becoming a medical home?

Probe Areas

Internal:

- QI experience

- People with experience and enthusiasm

- Adequate resources

External:

- Grant funds

- External training

-MDH certification

- NCQA recognition

4A. What barriers did you face in becoming a medical home?

4B. Can you tell me what you did to overcome those barriers?

4C. Were the actions you took successful in overcoming those barriers?

Probe Areas

- Teamwork or staff issues

- Work processes

- MDH standards/process

- Lack of reimbursement

- Lack of support from specialty consultants

Notes:
Probes

What do you mean?

Could you be more specific?

Could you say more about that?

Could you explain?

Anything else?

Any other comments?

What do you mean?

Could you be more specific?

Could you say more about that?

Could you explain?

Anything else?

Any other comments?

What do you mean?

Could you be more specific?

Could you say more about that?

Could you explain?

Anything else?

Any other comments?

What do you mean?

Could you be more specific?

Could you say more about that?

Could you explain?

Anything else?

Any other comments? 